\title{
Nonlinear Time-Domain Finite-Element Modeling of Thin Electromagnetic Shells
}

\author{
Ruth V. Sabariego ${ }^{1}$, Christophe Geuzaine ${ }^{1}$, Patrick Dular ${ }^{1,2}$ and Johan Gyselinck ${ }^{3}$ \\ ${ }^{1}$ Dept. of Electrical Engineering and Computer Science, University of Liège, Belgium \\ ${ }^{2}$ Fonds de la Recherche Scientifique - FNRS, Belgium \\ ${ }^{3}$ Dept. of Bio-, Electro- and Mechanical Systems (BEAMS), Université Libre de Bruxelles (ULB), Belgium
}

\begin{abstract}
A nonlinear time-domain extension of the classical linear frequency-domain thin-shell approach is presented. The interface conditions are expressed in terms of the average magnetic flux density throughout the shell thickness and a number of higher-order components. The method is elaborated in the frame of the magnetic vector potential formulation. The nonlinear system of algebraic equations is solved by means of the Newton-Raphson scheme. To validate the new formulation, we consider a magnetic plate placed above a double line carrying a sinusoidal current. Results are compared with those obtained with a fine finite-element model.
\end{abstract}

Index Terms-Electromagnetic shielding, thin-shell approximation, magnetodynamics, nonlinear time-domain analysis, finiteelement methods

\section{INTRODUCTION}

$\mathbf{T}$ HE USE of magnetic shields for mitigating the stray fields generated by electric and electronic devices becomes more and more important in order to comply with exposure limits and electromagnetic compatibility in particular in industrial environment. The finite-element (FE) analysis of these magnetic shielding problems involving thin shells may be computationally expensive and suffer from meshing difficulties due to the possibly large range of geometrical dimensions (from $\mathrm{mm}$ to $\mathrm{m}$ ) to handle.

These drawbacks can be overcome thanks to the thin-shell approach as the latter allows to reduce the thin-shell volume (thickness $d$ ) to an average surface situated halfway between its boundaries. However, it is most often restricted to linear and time-harmonic analyzes [1], [2], [3]. A nonlinear extension, combining a fixed-point iterative technique and a 1-D FE discretisation of the thin-shell surface, has been presented in [4]. This method still relies on a time-harmonic expression of the thin-shell boundary condition and obtains the time-domain solution via an inverse fast Fourier transform.

In [5], the authors propose a pure time-domain approach with the magnetic vector potential $(a-)$ formulation based on the use of dedicated even orthogonal polynomial basis functions to account for the variation of the even part of the magnetic flux and electric current density (linked, respectively, to the odd part of the electric current and magnetic flux density) through the shell thickness.

In this paper, the approach elaborated in [5] is extended and adapted to the nonlinear case. Even and odd parts of the magnetic flux density can no longer be uncoupled given the nonlinear nature of the problem. Therefore, the complete magnetic flux density is expanded using a set of both even and odd orthogonal basis functions. By way of validation, the thin-shell approach is applied to a 2-D problem: a magnetic plate placed above a double line carrying a sinusoidal current. Results are compared with those obtained with a fine finiteelement model.

Digital Object Identifier inserted by IEEE

\section{Magnetodynamic Formulation}

Let us consider a magnetodynamic problem in a bounded domain $\Omega=\Omega_{c} \cup \Omega_{c}^{C} \in \mathbb{R}^{3}$ with boundary $\Gamma$. The conductive and non-conductive parts of $\Omega$ are denoted by $\Omega_{c}$ and $\Omega_{c}^{C}$, respectively. Source inductors constitute domain $\Omega_{i} \subset \Omega_{c}^{C}$ (Fig. 1).

The Maxwell equations and constitutive laws governing the low-frequency eddy-current problems are

$$
\begin{gathered}
\operatorname{curl} h=j, \quad \operatorname{div} b=0, \quad \operatorname{curl} e=-\partial_{t} b, \\
h=h(b), \quad j=\sigma e,
\end{gathered}
$$

where $h$ is the magnetic field, $b$ the magnetic flux density (or induction), $e$ the electric field, $j$ the electric current density and $\sigma$ the conductivity (resistivity $\rho=1 / \sigma$ ).

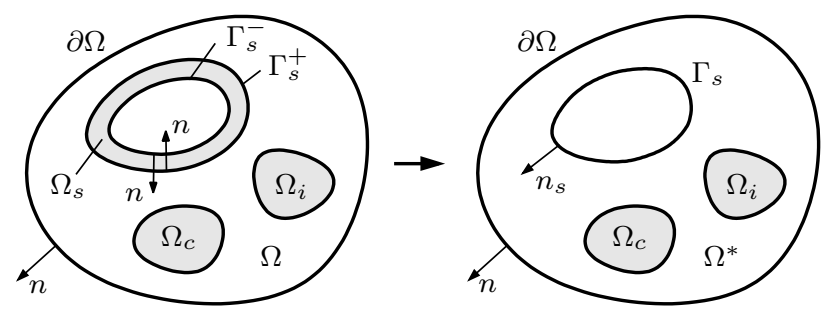

Fig. 1. Calculation domain $\Omega$ and reduction of the thin-shell domain $\Omega_{s}$ to the surface $\Gamma_{s}$

The $a$-formulation is obtained from the weak form of the Ampère law (1 a):

$$
\begin{array}{r}
\left(h(\operatorname{curl} a), \operatorname{curl} a^{\prime}\right)_{\Omega}+\left(\sigma \partial_{t} a, a^{\prime}\right)_{\Omega_{c}}+\left\langle n \times h, a^{\prime}\right\rangle_{\Gamma} \\
=\left(j_{i}, a^{\prime}\right)_{\Omega_{i}},
\end{array}
$$

where $a$ is the magnetic vector potential; $n$ is the outward unit normal vector on $\Gamma ; j_{i}$ is a prescribed current density; $(\cdot, \cdot)_{\Omega}$ and $\langle\cdot, \cdot\rangle_{\Gamma}$ denote a volume integral in $\Omega$ and a surface integral on $\Gamma$ of the scalar product of their arguments.

The first step in the thin-shell approach consists in reducing the thin-shell volume $\Omega_{s} \subset \Omega_{c}$ (thickness $d$ ) to an average surface $\Gamma_{s}$ situated halfway between the inner surface $\Gamma_{s}^{-}$and outer surface $\Gamma_{s}^{+}$of $\Omega_{s}$ (outward normal $n_{s}$ ), as depicted in Fig. 1. The surface integral in (2) is then modified on the basis of the 1-D thin-shell model described hereafter. 


\section{1-D THIN-SHELL MODEL}

In the 1-D model of the shell, only the variation of the magnetic field $h(z, t)$ and the magnetic induction $b(z, t)$ tangential to the boundary of the shell $\Gamma_{s}$ is considered throughout the shell thickness. We adopt a local coordinate system $x y z$ with the $z$-axis normal to the shell (i.e. parallel to $n_{s}$ ) and $z=0$ at its center. The tangential components of the magnetic field $h$ on $\Gamma_{s}^{+}$and $\Gamma_{s}^{-}$(both sides of the shell) are defined as:

$$
h_{t}^{+}=n_{s} \times\left(\left.h\right|_{\Gamma_{s}} ^{+} \times n_{s}\right), \quad h_{t}^{-}=n_{s} \times\left(\left.h\right|_{\Gamma_{s}} ^{-} \times n_{s}\right) \text {. }
$$

Analogously to (3), hereafter $f_{t}$ denotes the tangential component of a field $f$ on a surface $\Gamma$ with normal $n$.

\section{A. Governing differential equation}

The 1-D eddy-current problem in the shell $(-d / 2 \leq z \leq$ $d / 2)$ is governed by the following partial differential equation:

$$
\partial_{z}^{2} h_{t}(z, t)=\sigma \partial_{t} b_{t}(z, t)
$$

with nonlinear constitutive law $h=h(b)$.

The associated boundary conditions on the upper $(+)$ and lower (-) surfaces of the shell are given by

$$
h_{t}^{+}(t)=h_{t}(d / 2, t), \quad h_{t}^{-}(t)=h_{t}(-d / 2, t) .
$$

The average flux density vector $b_{0}(t)$ and the average current density vector $j_{0}(t)$, tangential to $\Gamma_{s}$, are essential global quantities. They read:

$$
b_{0}(t)=\frac{1}{d} \int_{-d / 2}^{d / 2} b_{t}(z, t) d z, \quad j_{0}(t)=\frac{1}{d} \int_{-d / 2}^{d / 2} j_{t}(z, t) d z .
$$

Further, taking into account (5) and the Ampère law (1 a), we have:

$$
h_{t}^{+}-h_{t}^{-}=-n_{s} \times d j_{0}(t) .
$$

\section{B. Linear harmonic case}

In the linear isotropic case, the constitutive law is $h=\nu b$, with $\nu$ the constant scalar reluctivity (permeability $\mu=1 / \nu$ ). For a sinusoidal time variation at pulsation $\omega$, we define the relative shell thickness as $d / \delta$, with $\delta=1 / \sqrt{2 / \sigma \mu \omega}$ the penetration depth.

Under these assumptions (4) can be solved analytically, which leads to an expression in terms of the complex representation (symbols in bold) of $h_{t}^{+}(t), h_{t}^{-}(t)$ and $b_{0}(t)$ [1]:

$$
\boldsymbol{h}_{t}^{+}+\boldsymbol{h}_{t}^{-}=2 \nu \boldsymbol{Y}(d / \delta) \boldsymbol{b}_{0},
$$

with

$$
\boldsymbol{Y}(d / \delta)=\frac{1+\boldsymbol{\imath}}{2} d / \delta \operatorname{cotanh}\left(\frac{1+\boldsymbol{\imath}}{2} d / \delta\right),
$$

where $\boldsymbol{\imath}$ is the imaginary unit. At low frequency, $0<d / \delta \ll 1$, $\boldsymbol{Y}$ tends towards 1; at sufficiently high frequency, say $d / \delta>6$, $\boldsymbol{Y}$ is practically equal to $\frac{1+\boldsymbol{\imath}}{2} d / \delta$.

The well-known FE frequency-domain approach includes the 1-D thin-shell model in a 2-D and 3-D analysis via the tangential fields $h_{t}^{+}, h_{t}^{-}$and (8) as done in [1], [2], [3].

\section{Nonlinear time-domain extension}

We now develop a time-domain extension of (8) by considering $n+1$ polynomial basis functions for the expansion of $b_{t}(z, t)$. In [5], the authors present a time-domain approximation of (8) based on an expansion of the even part of $b_{t}(z, t)$ with even orthogonal polynomial basis functions. The odd part of $b_{t}(z, t)$ is accounted for via the same kind of expansion for the even component of $j_{t}(z, t)$.

In this paper, the complete tangential induction $b_{t}(z, t)$ is expanded in terms of a set of orthogonal Legendre polynomials $\alpha_{k}(z)$, i.e. ,

$$
b_{t}(z, t)=\sum_{k=0}^{n} \alpha_{k}(z) b_{k}(t),
$$

where $\alpha_{0}=1, \alpha_{1}=2 z / d, \alpha_{2}=6 z^{2} / d^{2}-1 / 2, \alpha_{3}=$ $20 z^{3} / d^{3}-3 z / d, \cdots$ are normalized to verify $\left|\alpha_{k}( \pm d / 2)\right|=$ 1 , i.e. , their modulus equals one on either surface of the shell.

Strongly satisfying (4), the magnetic field $h_{t}(z, t)$ can thus be written as

$$
\begin{array}{r}
h_{t}(z, t)=\frac{h_{t}^{+}(t)}{+h_{t}^{-}(t)} \\
2 \\
+\sigma d^{2} \sum_{k=0}^{n} \beta_{k}(z) \partial_{t} b_{k}(t),
\end{array}
$$

where $d^{2} \partial_{z}^{2} \beta_{k}=\alpha_{k}(z)$ and $\beta_{k}( \pm d / 2)=0$.

Next, when considering a finite number of basis functions, the constitutive law $h(z, t)=h(b(z, t))$, whether linear or not, can be weakly impose as:

$$
\int_{-d / 2}^{d / 2} \alpha_{k}(z)\left(h_{t}(z, t)-h\left(b_{t}(z, t)\right)\right) \mathrm{d} z=0
$$

which leads to $n+1$ differential equations $(k=0, \ldots, n)$ in terms of $b_{0}(t), \ldots, b_{n}(t), h_{t}^{+}(t)$ and $h_{t}^{-}(t)$.

For the linear case, the following system of linear differential equations is obtained:

$$
[H(t)]=\nu[P][B(t)]+\sigma d^{2}[Q] \partial_{t}[B(t)],
$$

with $[H(t)]=\left[\begin{array}{lllll}\frac{h_{t}^{+}+h_{t}^{-}}{2} \frac{h_{t}^{+}-h_{t}^{-}}{6} & 0 & \cdots & 0\end{array}\right]^{T}$ and $[B(t)]=$ $\left[\begin{array}{llll}b_{0}(t) & b_{1}(t) & \cdots & b_{n}(t)\end{array}\right]^{T}$. The elements $p_{k}$ and $q_{k l}(k, l=$ $0, \ldots, n)$ of the diagonal matrix $[P]$ and triangular matrix $[Q]$ are given by:

$$
p_{k}=\int_{-d / 2}^{d / 2} \alpha_{k}(z) \alpha_{k}(z) \mathrm{d} z, \quad q_{k, l}=\int_{-d / 2}^{d / 2} \alpha_{k}(z) \beta_{l}(z) \mathrm{d} z .
$$

For example, with $n=1$, these values are $p_{0}=1, p_{1}=1 / 3$, $q_{00}=1 / 12, q_{01}=q_{10}=0$ and $q_{11}=1 / 60$.

In the nonlinear case, the system of algebraic equations (13) becomes

$$
[H(t)]=\int_{-d / 2}^{d / 2} h\left(b_{t}(t)\right)[A(z)] \mathrm{d} z+\sigma d^{2}[Q] \partial_{t}[B(t)],
$$

with $[A(z)]=\left[\alpha_{0} \cdots \alpha_{n}\right]^{T}$ and $b_{t}$ given by the expansion (10). The nonlinear algebraic equations that result from the time discretization of (15) can be solved by means of the Newton-Raphson method. With given $h_{t}^{+}(t)$ and $h_{t}^{-}(t)$, the Jacobian matrix reads

$$
\frac{\theta}{d} \int_{-d / 2}^{d / 2} \frac{\mathrm{d} h}{\mathrm{~d} b}[\Lambda] \mathrm{d} z+\frac{\sigma d^{2}}{\Delta t}[Q]
$$

where $\frac{\mathrm{d} h}{\mathrm{~d} b}$ is the differential reluctivity. The elements of matrix 
$[\Lambda]$ are the product of the basis functions $\alpha_{k}$, i.e. $\lambda_{k, l}=\alpha_{k} \alpha_{l}$ $(k, l=0, \ldots, n)$. The integration in the interval $[-d / 2, d / 2]$ can be done numerically by means of, e.g. , the 10-point Gauss scheme for the case $n=3$ [6].

\section{FE IMPLEMENTATION}

In the thin-shell formulation, the thin-shell volume $\Omega_{s}$ is excluded from the original calculation domain $\Omega$. Further, the surface $\Gamma_{s}$ with outward normal $n_{s}$ and situated halfway between the inner surface $\Gamma_{s}^{-}$and outer surface $\Gamma_{s}^{+}$of $\Omega_{s}$ is added to the new domain $\Omega \backslash \Omega_{s}$ (Fig. 1). In order to account for the changes in these domains, the surface integral term in (2) is modified. We consider that surfaces $\Gamma_{s}^{-}$and $\Gamma_{s}^{+}$have slightly moved so that they coincide with the average surface $\Gamma_{s}$. For the sake of simplicity, we abuse notation and keep on referring to them with the same names. The new weak form reads:

$$
\begin{gathered}
\left(h(\operatorname{curl} a), \operatorname{curl} a^{\prime}\right)_{\Omega \backslash \Omega_{s}}+\left(\sigma \partial_{t} a, a^{\prime}\right)_{\Omega_{c}}+\left\langle n \times h, a^{\prime}\right\rangle_{\Gamma} \\
+\left\langle n_{s} \times h, a^{\prime}\right\rangle_{\Gamma_{s}^{-}}-\left\langle n_{s} \times h, a^{\prime}\right\rangle_{\Gamma_{s}^{+}}=\left(j_{i}, a^{\prime}\right)_{\Omega_{i}} .
\end{gathered}
$$

The time-domain behavior of the thin shell is taken into account by introducing the tangential vector fields $b_{0}, b_{1}, \cdots b_{n}$ on $\Gamma_{s}$ as unknowns.

The tangential component of the magnetic vector potential $a_{t}$ is discontinuous across $\Gamma_{s}$ and is related to the net flux $d b_{0}$ in the shell as

$$
a_{t}^{+}-a_{t}^{-}=-n_{s} \times d b_{0}(t) .
$$

We therefore decompose $a$ as $a_{c}+a_{d}$, the tangential components of $a_{c}$ and $a_{d}$ being continuous and discontinuous across the shell, respectively.

For $a_{c}$ a conventional discretization with Whitney elements is adopted. Without loss of generality we can choose $a_{d}$ to be zero in the volume enclosed by $\Gamma_{s}$. Furthermore, conformity can be ensured by limiting its support to one layer of elements touching $\Gamma_{s}^{+}$[3]. By considering $a^{-}=a_{c}$ and $a_{d}=-n_{s} \times$ $d b_{0}$ together with (7), we can work out the two new surface terms in (2). They are given by

$$
\begin{aligned}
& \left\langle n_{s} \times h, a^{\prime}\right\rangle_{\Gamma_{s}^{-}}-\left\langle n_{s} \times h, a^{\prime}\right\rangle_{\Gamma_{s}^{+}} \\
& =-\left\langle n_{s} \times h_{t}^{+}, a_{c}^{\prime}\right\rangle_{\Gamma_{s}}-\left\langle n_{s} \times h_{t}^{+}, a_{d}^{\prime}\right\rangle_{\Gamma_{s}}+\left\langle n_{s} \times h_{t}^{-}, a_{c}^{\prime}\right\rangle_{\Gamma_{s}} \\
& =d\left\langle h_{t}^{+}, b_{0}^{\prime}\right\rangle_{\Gamma_{s}}-d\left\langle j_{0}, a_{c}^{\prime}\right\rangle_{\Gamma_{s}},
\end{aligned}
$$

where $j_{0}(6 \mathrm{~b})$ is obtained from (1 a) and (11).

In the linear case, using the first two lines of system (13) we get an expression for $h_{t}^{+}$and $h_{t}^{-}$in terms of $b_{0}, b_{1}, b_{2}$ and $b_{3}$ (assuming $n \geq 2$ ), i.e.

$$
\begin{aligned}
h_{t}^{ \pm} & =\nu b_{0}+\sigma d^{2}\left(q_{00} \partial_{t} b_{0}+q_{02} \partial_{t} b_{2}\right) \\
& \pm 3 \nu b_{1} \pm 3 \sigma d^{2}\left(q_{01} \partial_{t} b_{1}+q_{03} \partial_{t} b_{3}\right) .
\end{aligned}
$$

The weak form (17) is thus coupled with the time-domain thin-shell approximation via $a_{c}, a_{d}$ in $\Omega \backslash \Omega_{s}$ and $b_{0}, b_{1}, b_{2}$ and $b_{3}$ on $\Gamma_{s}$.

Next, from (7) and applying the Ampère law (1 a) to (11), we get the second condition concerning the tangential components $a_{c, t}$ and $a_{d, t}$ of $a_{c}$ and $a_{d}$. We have:

$$
-\sigma \partial_{t}\left(2 a_{c, t}+a_{d, t}\right) / 2=\frac{2}{d} \nu b_{1}+\sigma d\left(\frac{1}{5} \partial_{t} b_{1}-\frac{1}{70} \partial_{t} b_{3}\right)
$$

which we can weakly imposed on $\Gamma_{s}$ with test functions $b_{1}^{\prime}$ and $b_{3}^{\prime}$.

The remaining equations of system (13) result in the following weak forms with test functions $b_{l}^{\prime}(l=2,3, \cdots, n)$ :

$$
0=\left\langle\nu p_{l} b_{l}, b_{l}^{\prime}\right\rangle_{\Gamma_{s}}+\sum_{i=-2,0,2}\left\langle\sigma d^{2} q_{l, l+i} \partial_{t} b_{l+i}, b_{l}^{\prime}\right\rangle_{\Gamma_{s}} .
$$

In the nonlinear case, the local vectors $h_{t}$ and $b_{t}$ in the thin shell are linked through the nonlinear relation $h=h(b)$. The nonlinearity affects thus only the terms $\nu b_{0}, \nu b_{1}, \nu b_{l}$ in (20), (21) and (22). The Jacobian matrix (16) holds also for these terms, what allows to apply the Newton-Raphson method to solve the system of nonlinear algebraic equations obtained after space and time discretization of (17) and (15).

\section{Application ExAMPLE}

The proposed thin-shell approach is validated by means of a $2 \mathrm{D}$ application example, and up to $n=5$. We consider a magnetic and conducting thin plate (length $1 \mathrm{~m}, d=1 \mathrm{~mm}$, $\sigma=11.11 \mathrm{MS} / \mathrm{m}$ ) placed $10 \mathrm{~cm}$ above a double line carrying a sinusoidal current of $5250 \mathrm{~A} \mathrm{rms}$ (the distance between the conductors is $30 \mathrm{~cm}$ ), see Fig. 2. A nonlinear $B H$-relation is considered for the thin shell, viz $h(b)=100 b+10 b e^{1.8 b^{2}}$ with $h$ in $\mathrm{A} / \mathrm{m}$ and $b$ in $\mathrm{T}$.

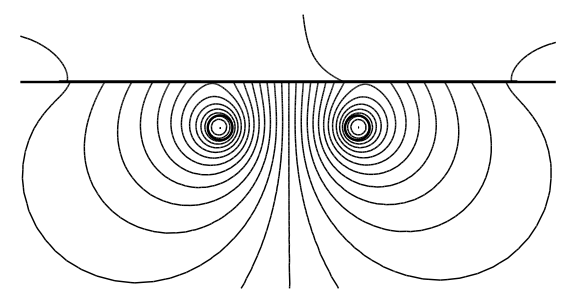

Fig. 2. Magnetic and conducting plate placed above a double line and flux lines at $d / \delta=2$

A brute-force FE model with a fine discretization of the thin-shell (number of layers of elements equals $3 \max (d / \delta, 1)$ ) provides a reference solution, $\delta$ being the penetration depth for a linear material $\left(\mu_{r}=1000\right.$ corresponding to $\left.b=1.5 \mathrm{~T}\right)$. Results inside and outside the plate are compared.

Time-stepping simulations with imposed sinusoidal current of same amplitude but three different frequencies are carried out, namely $f=11.8 \mathrm{kHz}(d / \delta=1), f=189.75 \mathrm{kHz}(d / \delta=$ $2)$ and $f=961.53 \mathrm{kHz}(d / \delta=3)$. One period $T=1 / f$ and a half $[0,3 T / 2]$ is time-stepped with $\Delta t=T / 120$. The system of nonlinear algebraic equations is solved by means of the Newton-Raphson method.

The variation of the flux throughout the thickness of the shell at $25 \mathrm{~cm}$ of its center is evidenced in Fig. 3 with $d / \delta=1$ and for two different time-steps. The approximation improves clearly with $n$, though more terms $b_{n}$ are needed for increasing the precision inside the plate. As shown in Figs. 5 and 6, the estimation is sufficient for ensuring a good accuracy of the average flux in the plate and the flux outside the plate.

The average magnetic flux in the plate is shown in Fig. 4. An excellent agreement is observed between flux waveforms obtained with the fine model and the thin-shell model for 


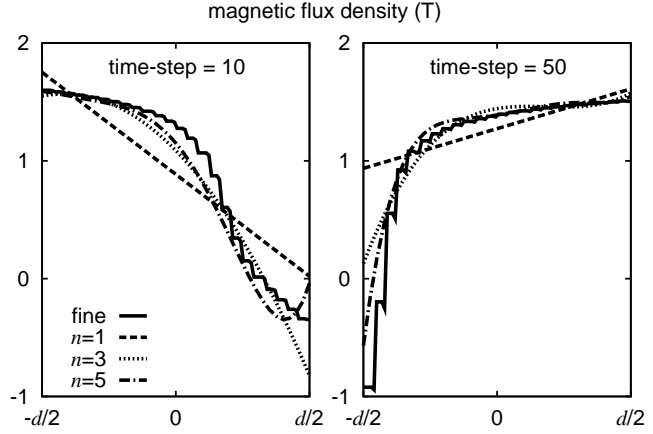

Fig. 3. Variation of the induction throughout the thickness of the shell at $25 \mathrm{~cm}$ of its center with $d / \delta=1$ obtained with the fine model and the thinshell approach

$d / \delta=1,2,3$. The higher the ratio $d / \delta$, the higher the number of additional terms in the expansion of $b_{t}$ required. One clearly observes the saturation and the effect of the eddy currents.

Results for a point situated at $0.125 \mathrm{~m}$ above the center of the plate are depicted in Figs. 5 and 6. The agreement between the curves is very satisfactory for the given $n$. The solution obtained with the thin-shell approach converges quickly to the reference solution when increasing $n$, as evidenced in Fig. 6.

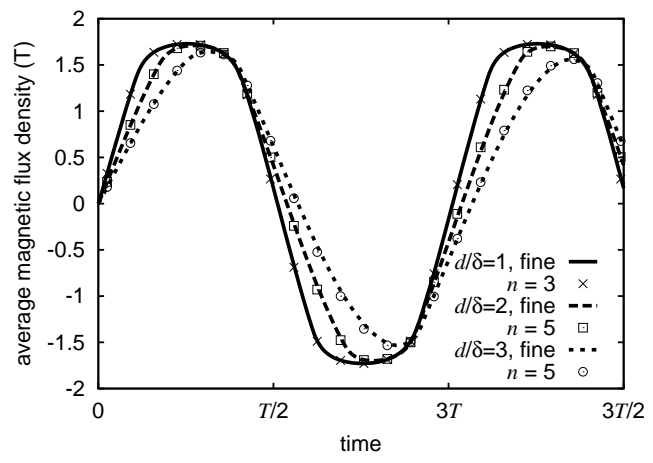

Fig. 4. Average magnetic flux density in the plate versus time with $d / \delta=$ $1,2,3$. Results computed with the fine model and the thin-shell approach. Value of $n$ ensuring a good agreement

\section{CONCLusions}

A new nonlinear time-domain finite-element method for the analysis of thin-shells has been elaborated. The proposed method is based on the coupling of a nonlinear time-domain 1-D thin-shell model with a magnetic vector potential formulation via the surface integral term. A number of additional unknowns for the magnetic flux density are incorporated on the shell boundary. The nonlinear system is solved by means of the Newton-Raphson scheme.

A clear advantage of the proposed thin-shell approach is that the mesh of the computation domain does not depend on the working frequency anymore. Furthermore, for a given accuracy, when increasing the frequency, the number of additional unknowns is very limited in comparison with those required by a classical $\mathrm{FE}$ model, what is specially interesting in cumbersome 3-D problems. It provides a good compromise

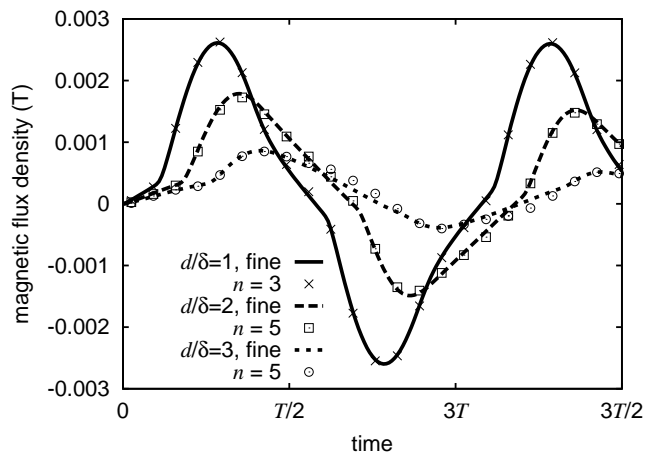

Fig. 5. Magnetic flux density in a point situated at $0.125 \mathrm{~m}$ above the center of the plate versus time with $d / \delta=1,2,3$. Results computed with the fine model and the thin-shell approach. Value of $n$ ensuring a good agreement

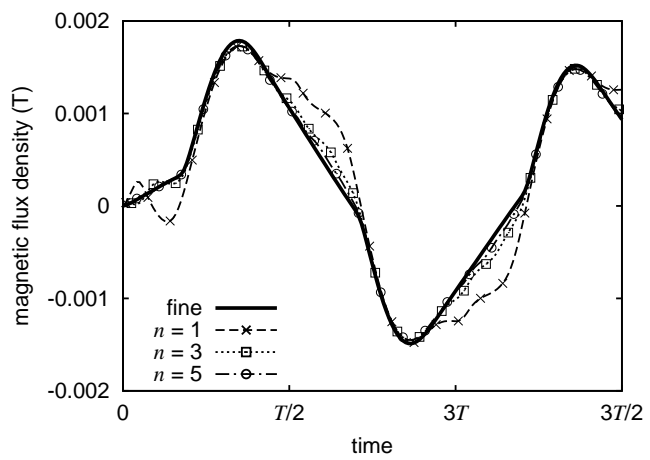

Fig. 6. Magnetic flux density in a point situated at $0.125 \mathrm{~m}$ above the center of the plate versus time with $d / \delta=2$. Results computed with the fine model and the thin-shell approach $(n=1,3,5)$

between computational cost and accuracy. Indeed, adding a sufficiently large number of induction components in the thin shell, a very high accuracy can be achieved.

\section{ACKNOWLEDGMENT}

This work was partly supported by the Belgian Science Policy (IAP P6/21).

\section{REFERENCES}

[1] L. Krähenbühl and D. Muller, "Thin layers in electrical engineering. example of shell models in analyzing eddy-currents by boundary and finite element methods," IEEE Trans. on Magn., vol. 29, no. 5, pp. 14501455, 1993.

[2] I. D. Mayergoyz and G. Bedrosian, "On calculation of 3-D eddy currents in conducting and magnetic shells," IEEE Trans. on Magn., vol. 31, no. 3, pp. 1319-1324, 1995.

[3] C. Geuzaine, P. Dular, and W. Legros, "Dual formulations for the modeling of thin electromagnetic shells using edge elements," IEEE Trans. on Magn., vol. 36, no. 4, pp. 799-803, 2000.

[4] O. Bottauscio, M. Chiampi, and A. Manzin, "Transient analysis of thin layers for the magnetic field shielding," IEEE Trans. on Magn., vol. 42, no. 4, pp. 871-874, 2006.

[5] J. Gyselinck, R. V. Sabariego, P. Dular, and C. Geuzaine, "Time-domain finite-element modelling of thin electromagnetic shells," IEEE Trans. on Magn., vol. 44, no. 6, pp. 742-745, June 2008.

[6] J. Gyselinck, R. V. Sabariego, and P. Dular, "A nonlinear time-domain homogenization technique for laminated iron cores in three-dimensional finite element models," IEEE Trans. on Magn., vol. 42, no. 4, pp. 763 766, 2006.

Manuscript received May 10, 2008. Corresponding author: Ruth V. Sabariego (R.Sabariego@ulg.ac.be). 